\title{
Peripheral visual processing
}

\author{
DEBORAH LOTT HOLMES \\ Loyola University, Chicago, Illinois 60626 \\ KAREN M. COHEN \\ Harvard University, Cambridge, Massachusetts 02138 \\ MARSHALL M. HAITH \\ University of Denver, Denver, Colorado 80210 \\ and \\ FREDERICK J. MORRISON \\ Dartmouth College, Hanover, New Hampshire 03755
}

\begin{abstract}
An attempt was made to examine the development of the ability to identify stimuli presented to peripheral vision in several different tasks. Five- and 8-year-old children and college adults saw, for $20 \mathrm{msec}$, either a single figure at $1^{\circ}, 2^{\circ}, 4^{\circ}$, or $6^{\circ}$ of visual angle from the fovea (singleform condition) or an off-foveal figure with an additional figure at the fovea (double-form condition). In the double-form conditions, the subjects were required to identify either the peripheral figure only (double-form presentation) or both figures (double-form report). The main effects of Age, Distance, and Form Condition were significant. Accuracy improved with increasing age and with decreasing distance. The Form Condition effect reflected lower accuracy in the two double-form conditions than in the single-form condition. Interestingly, there was no difference between the two double-form conditions, suggesting that the mere presence of a foveal stimulus, with instructions to ignore it, produces as much decrement in peripheral performance as when subjects are told to fully process and report the foveal stimulus. Also, there was no interaction between Form Condition and Distance, suggesting that the label "tunnel vision" may be misleading, since the presence of the foveal stimulus seems to have an equal effect on all peripheral locations and does not really "restrict" the size of the effective visual field.
\end{abstract}

Visual events are perceived not only in the area of central, or foveal, vision, but in the area of peripheral vision as well. Some of these peripherally occurring events may be detected, fully processed, and identified by the observer without the necessity of eye movements. Other peripheral events may be detected and perhaps partially processed, but not completely identified. In these latter cases, the observer may move his eyes in order to place the

The authors wish to acknowledge the valuable assistance of the people involved in this research. We are grateful to Richard Hansen and the teachers at Eastridge Elementary School for their cooperation in providing children. Deborah Porter and Celeste Newman deserve special thanks for their assistance in data collection; Frank Slaymaker and Lynne Olsho provided invaluable suggestions and help in data analysis. This research was supported by a research grant from Loyola University to Deborah Holmes and by a grant from the National Institute of Child Health and Human Development (HD0723202) to Marshall Haith. A version of this paper was presented at the meetings of the Society for Research in Child Development held in New Orleans, March 1977. Requests for reprints should be sent to Deborah Holmes, Psychology Department, Loyola University of Chicago, 6525 North Sheridan Road, Chicago, Illinois 60626. peripheral events onto the more sensitive fovea for detailed examination.

A growing body of research has examined information processing in the periphery in the absence of eye movements. Several variables have been found to affect whether or not a stimulus in peripheral vision can be identified without the use of eye movements which bring it into foveal vision. One variable is whether or not other stimuli are present in the visual field: subjects are less able to identify a peripheral stimulus when other stimuli are simultaneously presented than when the peripheral stimulus is presented alone (Ikeda \& Takeuchi, 1975). Similarly, when subjects are asked to follow a centrally located fixation dot (Leibowitz \& Appelle, 1969 ) or to count the number of fixation points presented foveally (Webster \& Haslerud, 1964), visual processing of peripherally located stimuli suffers.

In all of these experiments, the effective visual field (or that portion of space in which a peripherally located stimulus can be detected) shrinks when subjects are asked to perform some kind of analysis 
on simultaneously presented stimuli. This taskinduced shrinkage of the effective visual field has been called "tunnel vision" (Mackworth, 1965). Investigators have generally assumed that it is the actual processing of the additional stimuli, rather than their mere presence, which interferes with the subject's ability to visually process the peripheral stimuli. This interpretation is, however, not completely satisfactory, since Mackworth (1965) found that the mere presence of additional irrelevant stimulus items in the visual field produced a similar interference in the subject's ability to detect similarities among foveally and peripherally presented stimuli. However, Mackworth's study left open the question of whether requiring subjects to fully analyze these additional stimuli would have further restricted the size of the effective visual field.

A second variable which may affect the size of the effective visual field is the age of the subject. Perceptual research with young children suggests that they use peripheral vision information less efficiently than do adults in guiding future eye movements. Thus, the average eye-movement latency to a peripheral stimulus is almost twice as long for young children as it is for adults (Miller, 1969), and children's eye movements when inspecting a picture are about onethird shorter than adults' (Mackworth \& Bruner, 1970). However, these studies are concerned with the child's motor response patterns (i.e., his eye movements) to peripheral stimulation and provide limited insight into children's relative ability to identify peripheral stimuli.

At least three studies (Lakowski \& Aspinall, 1969; Miller, 1971; Whiteside, 1976) have specifically investigated developmental changes in the ability to detect peripheral visual information. The first study (Lakowski \& Aspinall, 1969) used standard perimetric methods and revealed marked deficiencies in the abilities of young children to detect peripheral stimuli. This first study is difficult to interpret, however, since it is possible that children may use a stricter criterion for making a detection response than do adults. Whiteside (1976) eliminated this problem in a later study by measuring the threshold luminance of the peripheral stimulus necessary to evoke an eye movement instead of relying on subjects' verbal reports; he found no age differences in peripheral sensitivity to light flashes in subjects aged 6 to 21 years. Similarly, Miller (1971) failed to demonstrate a more rapid decrease in detectability of stimuli for 2nd grade children, as compared to 6th graders and adults, as the stimulus was presented at increasing distances from the fovea. However, Miller's study did not provide a truly adequate test of age differences in peripheral processing, since the greatest distance from the fovea studied was only $2.5^{\circ}$, and the youngest age group studied was 7-year- olds. Two additional studies (Holmes, 1972) investigated the ability of 5 - and 8 -year-old children and adults to identify visual stimuli presented at varying distances from the fovea. These studies suggested that, under conditions where a single stimulus of some complexity or multiple stimuli are presented, young children have greater difficulty with peripherally presented stimuli than do adults. In sum, then, developmental studies of peripheral vision have produced contradictory results.

The main purpose of the present study was to investigate the hypothesis that the effective visual field of 5-year-old children is smaller than that of older children and adults. In addition, we examined the questions of whether task demands affect the size of the effective visual field (i.e., "tunnel vision") and whether or not these task demands affect the performance of young children and adults differentially. The experiment therefore examined performance in three conditions of increasing difficulty: identification of a single geometric form located peripherally; identification of a peripheral form presented simultaneously with a foveally located form which was to be ignored; and identification of a peripheral form presented with a foveally located form, both of which were to be reported.

\section{METHOD}

\section{Subjects}

A total of 54 subjects were studied (18 5-year-olds, 18 8-yearolds, and 18 adults). All of the children were enrolled at Eastridge Elementary School in Denver, Colorado. The adult sample consisted of undergraduates at the University of Denver. All subjects had normal uncorrected vision.'

\section{Materials}

All stimuli were constructed from black outline figures of eight possible simple geometric forms (circle, line, arrow, square, star, triangle, " $x$," and sideways " $S$ ") on a white background. The figures were decals produced by Chartpak Rotex. Each figure subtended 33' of visual angle, with a line thickness of approximately $8^{\prime}$ of visual angle.

In the single-form condition, each stimulus card contained one of the geometric forms centered at one of four distances from the fovea (approximately $1^{\circ}, 2^{\circ}, 4^{\circ}$, or $6^{\circ}$ ) and along one of eight possible radii from center (top, bottom, left, right, and diagonals). There were eight stimulus cards for each of the four distances studied: within these eight cards, each form and each radial position was represented exactly once. Moreover, for each distance, different combinations of forms and radial positions were employed. In all other respects, the assignment of stimuli and positions to trials was random. Two separate sets (of eight stimuli per four distances) were generated in the above manner in order to minimize any confounding of forms and radial position with distance.

In the double-form conditions, the same stimulus materials were used as in the single-form condition, with the exception that a second geometric form was added to the center of each stimulus card. The selection of this "foveal" form was random, with the restriction that it never be identical to the form presented to the periphery. 


\section{Apparatus}

A Gerbrands' four-field tachistoscope was used. Field illumination was $10.28 \mathrm{~cd} / \mathrm{m}^{2}$ for both the fixation and stimuius fields. Viewing distance was $81.22 \mathrm{~cm}$.

\section{Procedure}

Three experimental form conditions were employed. The first form condition (single form) required verbal identification of the geometric form which appeared at one of the peripheral distances studied. In the second form condition (double-form presentation), two geometric forms were presented on each trial: one form presented at the center of the field and the other at one of the peripheral distances studied. Subjects were instructed to ignore the foveal stimulus and to report only the peripheral stimulus. The last form condition (double-form report) was identical to the double-form presentation condition, except that subjects were required to report both the peripheral and foveal stimuli. Forcedchoice verbal reports were used to eliminate any possible age differences in the tendency to say "I don't know."

All of the testing was conducted by three female experimenters, who worked in teams of two. One experimenter sat beside the subject, giving instructions, recording responses, and providing feedback on performance; the other experimenter was behind the tachistoscope and changed stimulus cards and exposure durations.

Each child was tested in three separate sessions (the first of which served as a practice session and was not included in data analysis), lasting approximately $20 \mathrm{~min}$ each. These sessions were completed within 3 to 5 days for each subject. Adults were tested in one session divided into three parts, with 5 -min breaks between them. As with the children, adult data from the first part of the session was excluded from analysis. The total time for the adult testing session was $30 \mathrm{~min}$. The subjects were assigned randomly to conditions and to one of the two stimulus sets within each condition.

At the beginning of each session, the subject was shown a card representing the eight different geometric forms employed as stimuli and asked to label them. This preliminary procedure was employed to insure that all subjects were familiar with the stimuli and that they had readily available labels for them. During each session, each subject received a total of 34 trials. Eighteen of the trials were the experimental trials described earlier. The subjects were not informed as to whether a particular trial was experimental or practice. The sequence of trials during the last two sessions was as follows: Two practice trials each at 150-, 40-, and 30-msec exposure durations for stimuli located at $1^{\circ}$ of visual angle from a center fixation dot. Then there were three practice trials at $20 \mathrm{msec}$ (the exposure duration at which all of the temaining trials were presented) at $1^{\circ}$, followed by four experimental trials at $1^{\circ}$. Next, there were three practice trials followed by four experimental trials at $2^{\circ}$, at $4^{\circ}$, and at $6^{\circ}$ of visual angle. The rationale for using such massive practice procedures was to avoid learning effects. Only the ascending order of the stimulus distance $\left(1^{\circ}, 2^{\circ}, 4^{\circ}, 6^{\circ}\right)$ was used, because pilot work (Holmes, 1972) had indicated that young children found the task too difficult when a nonascending series was used.

On an experimental trial, the following sequence of events transpired. The subject pushed a starter button while fixating a dot in the center of an illuminated field. Seven hundred and fifty milliseconds later, the stimulus flashed for $20 \mathrm{msec}$. The subject then verbally reported what he had seen, except in the double-form presentation condition, where the subjects were instructed to report only the stimulus that was "farther away." All subjects were given veridical feedback, and the children were given a poker chip for each correct response. At the end of each session, the children traded in their poker chips for a piece of candy. Adult subjects were given credit for the introductory psychology subjectpool requirement.

\section{Design}

There were three experimental form conditions: (1) the singleform condition, (2) the double-form presentation condition, and (3) the double-form report condition. For each condition, there were two sets of trials with different combinations of stimuli and positions. Each subject saw only one set of stimuli for one of these experimental conditions. Each subject received a total of 32 experimental trials distributed equaliy over two experimental sessions. Within each session, each subject had the following sequence of experimental trials; four trials at $1^{\circ}$, four trials at $2^{\circ}$, four trials at $4^{\circ}$, and four trials at $6^{\circ}$. In addition, there were three age groups: 5-year-olds, 8-year-olds, and adults. In summary, a mixed design was used, with Age, Set, and Form Condition as between-subjects variables and Distance as a within-subjects variable.

\section{RESULTS}

The scores for each subject's performance were corrected for guessing ${ }^{2}$ and subjected to a four-way analysis of variance, with Distance as a within: subjects variable, and with Age, Form Condition, and Set as between-subjects variables. There was a significant main effect for Age, $F(2,36)=14.45$, $\mathrm{p}<.01 .^{3}$ Orthogonal comparisons indicated that 5 -year-olds and 8-year-olds did not differ reliably, $F(1,108)=1.74, p>.10$, but the two groups of children combined differed clearly from adults, $\mathrm{F}(1,108)=5.49, \mathrm{p}<.05$. There was a significant main effect for Distance, $F(3,108)=124.59, p<.01$ : performance dropped steadily as the stimulus distance increased from $2^{\circ}$ to $6^{\circ}$ of visual angle. There was also a significant main effect for Form Condition, $F(3,36)=5.47, p<.01$; performance was highest in the single-form condition, followed by the double-form presentation condition, and finally the double-form report condition. (Mean corrected accuracy was $7.00,6.63,6.48$, respectively.) Orthogonal comparisons failed, however, to reveal significant differences among the different form conditions. The comparison of the two double-form conditions was $F(1,108)=0.16, p>.25$; the comparison of the two double-form conditions vs. the single-form condition was $F(1,108)=2.63$, p > .10 .

There was a significant interaction between Age and Distance, $F(6,108)=2.58, p<.05$, with the performance of the 5- and 8-year-olds falling off more rapidly than the performance of the adults as a function of increased distance from the fovea. However, even the 5-year-olds performed quite well (better than $50 \%$ accuracy after corrections for guessing) at the farthest peripheral distance studied.

There was also an unexpected significant Set by Form Condition interaction, $F(2,36)=6.21, p<.01$. No obvious explanation is available for this particular finding.

The predicted Form Condition by Distance inter- 
action failed to achieve statistical significance, although it was close, $F(6,108)=1.89, p<.10$. Similarly, the predicted Age by Form Condition by Distance interaction was statistically insignificant, $F(12,108)=0.76, p>.25$.

Several planned orthogonal comparisons were executed on the condition levels at $6^{\circ} .{ }^{4}$ For adults, there was a significant difference between the singleform condition and the two double-form conditions combined, $F(1,36)=5.50, \mathrm{p}<.05$. The difference between the two double-form conditions, however, failed to achieve statistical significance, $F(1,36)=$ $0.46, p>.25$. Thus, the presence of a second foveal stimulus interfered with the adults' ability to identify a distant peripheral stimulus regardless of whether they actually were required to process the foveal stimulus.

For 8-year-olds, there was also a significant difference between the single-form condition and the two double-form conditions combined, $F(1,36)=12.39$, $\mathrm{p}<.01$. Like the adult data, the performance of the 8 -year-olds did not differ reliably between the two double-form conditions, $F(1,36)=0.46, p>.25$. Thus, as for adults, the mere presence of a foveal item interfered with the performance of the 8-yearolds on the more distant stimulus.

For the 5-year-old subjects, neither of the comparisons of $6^{\circ}$ (single-form vs. double-form conditions, and double-form presentation vs. double-form report) revealed a difference among the different conditions, $F(1,36)=0.34, p>.25$, and $F(1,36)$ $=1.03, \mathrm{p}>.25$. Thus, neither the number of the stimuli nor the assumed difficulty of the particular tasks differentially affected the performance of the youngest children at $6^{\circ}$.

As is apparent in Figures 1 and 2, the data were characterized by the presence of ceiling effects at $1^{\circ}$. Since these ceiling effects resulted in nonhomogeneity of variance, the data were reanalyzed using only data from $2^{\circ}, 4^{\circ}$, and $6^{\circ}$ of visual angle. The results of this second analysis are quite similar to those of the analysis just described. Significant main effects were: Age, $F(2,36)=13.71$, $p<.01$; Form Condition, $\mathrm{F}(2,36)=4.41, \mathrm{p}<.05 ;$ and Distance, $\mathrm{F}(2,72)=$ $106.09, \mathrm{p}<.01$. The only significant interaction, however, was that of Form Condition by Set, $F(2,36)$ $=6.41, p<.01$. The Age by Distance interaction revealed in the earlier analysis was not significant when the data were truncated in this manner, $F(4,72)$ $=0.82, p>.10$. As in the earlier analyses, the Distance by Form Condition interaction was not significant, $F(4,72)=1.85, p>.10$. The results of the orthogonal comparisons were also unchanged by this truncation of the data.

\section{DISCUSSION}

The results of this study are best understood by examining the effects of the three main variables:

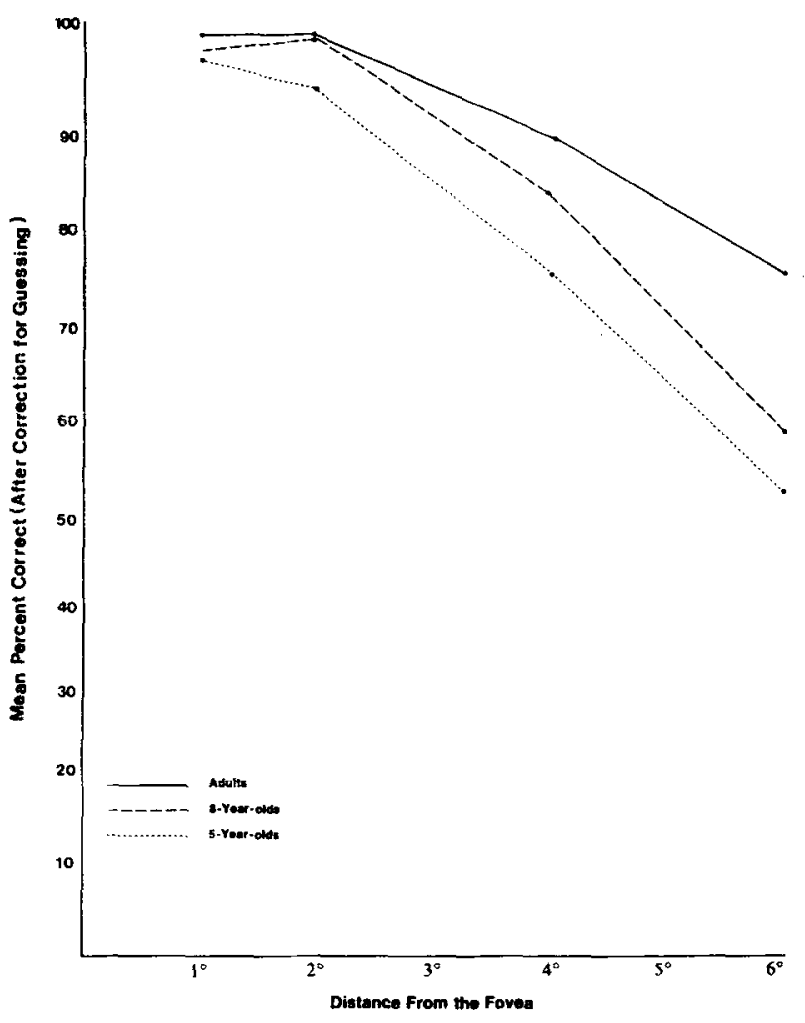

Figure 1. Mean accuracy for each age group at $1^{\circ}, 2^{\circ}, 4^{\circ}$, and $6^{\circ}$ of visual angle.

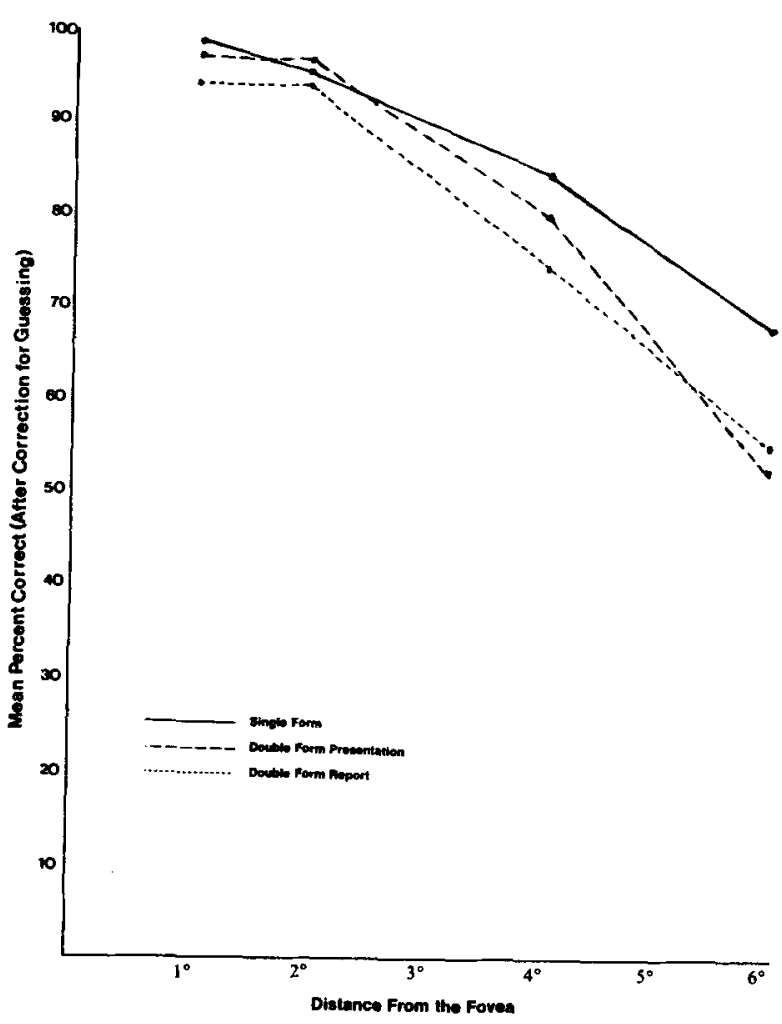

Figure 2. Mean accuracy for each condition at $1^{\circ}, 2^{\circ}, 4^{\circ}$, and $6^{\circ}$. 

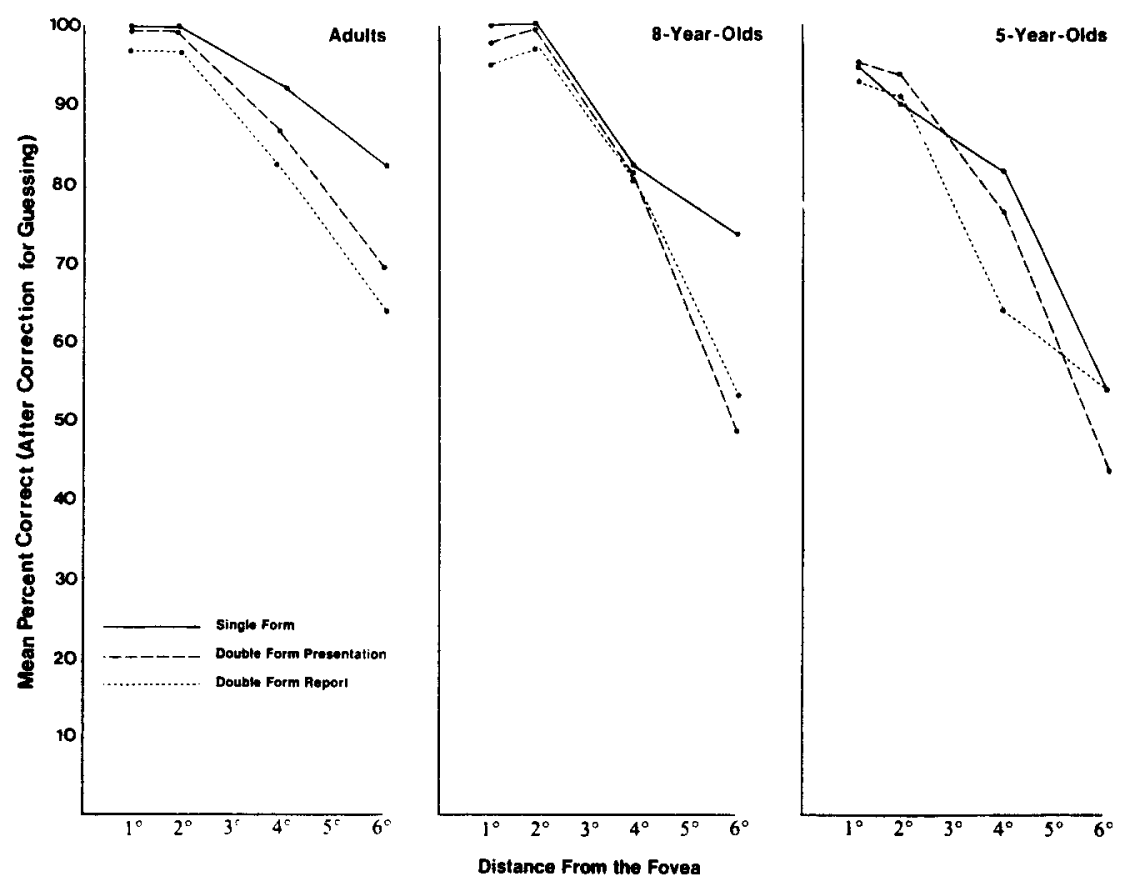

Figure 3. Mean accuracy for each age group as a function of distance from the fovea and experimental condition.

distance, age, and form condition. All three of these variables had a significant effect on performance, and there is some indication that their effects interact in fairly complex ways.

First, consider the extent to which the performance of subjects differed as a function of their age. Performance was significantly higher for adults than for the 5- and 8-year-olds. However, the finding of a significant main effect for age is rather uninformative concerning peripheral visual processing. The children may have performed more poorly simply because they had poor motivation or did not maintain attention as well as adults. If the generally poorer performance of the children of this study involves their ability to perceive peripheral stimuli (rather than reflecting motivational and attentional factors), then one would predict that, as stimuli were presented at increasingly greater distances from the fovea, performance of the children would be increasingly affected. In other words, performance should have dropped more rapidly with distance for children than for adults, and have resulted in the presence of an interaction between age and distance. There was, however, only weak evidence for the presence of such an interaction.

The clearest and most interesting finding of this study is the effect of the different form conditions on performance. Performance was highest when the subjects saw only a single stimulus item located peripherally. The two conditions where subjects simultaneously saw both foveal and peripheral items produced poorer performance. Moreover, requiring subjects to actually process and report the foveal stimulus had relatively little impact on performance with the peripheral stimulus, although it did produce a slight reduction in this performance. Thus, it appears that the typical findings that central tasks interfere with peripheral visual performance are probably better interpreted in terms of the presence of the foveal stimuli than in terms of the requirement that the foveal stimuli be reported.

The study also suggests possible answers to the key question of why the simultaneous presence of a foveally presented item interferes with identification of a peripheral item. It is generally assumed that the presence of the central item may somehow restrict the size of the visual field. This "restricted visual field" or "tunnel vision"' argument implies that there should be an increasing effect of the foveal item on identification of the peripheral item with increasing distance. An alternative hypothesis is that the foveal item somehow draws the attention of the observer and, therefore, interferes with the processing of all other stimulus items in the field, regardless of their location. In other words, subjects may actually fully process the foveal items, regardless of whether or not they have been instructed to do so. If this "general interference" hypothesis is correct, then the presence of the foveal item should have a fairly constant interference effect across all peripheral stimulus locations. The absence in our data of a significant interaction between Form Condition and Distance suggests that the general interference explanation may be the more tenable of the two. 
A question of concern in this study was whether or not age is an important factor in determining the effects of the foveal item on performance. The results of the planned orthogonal comparisons performed on the data obtained at $6^{\circ}$ of visual angle are relevant to this question. These comparisons were limited to the $6^{\circ}$ data, since this was the greatest peripheral distance studied, and therefore should have produced the most pronounced effects of form condition. These comparisons revealed that the different form conditions affected performance with peripheral items somewhat differently for the three age groups. Although neither the number of stimulus items present nor the number of items to be reported affected the performance of the 5-year-olds, the number of stimulus items in the display did affect the performance of the 8-year-olds and the adults.

In particular, for the 8-year-olds and the adults, the presence of a foveal item interfered with identification of the peripheral item regardless of whether or not actual processing of the foveal item was necessary. This finding is interpreted as evidence that adults and older children may have been "locked in" to a processing strategy that directed them to attend to stimulus items from the center out, whether or not they had instructions to do so. This interpretation would argue as follows: when only a single item was presented to peripheral vision, performance was high, as total attention was directed immediately to it. When two items were simultaneously presented, the center one was attended first (even if it was irrelevant), so that only a fading trace of the rapidly decaying peripheral item remained by the time attention was directed to it. This interpretation is supported by an examination of the order in which items were reported in the double-form report condition. For both the 8-year-olds and the adults, there was a definite tendency to report the foveal items first: only $21 \%$ and $14 \%$ of the correctly reported peripheral items were reported first by the 8-year-olds and the adults, respectively. This explanation also seems quite reasonable in light of the fact that the overall effect for form condition was relatively constant and did not increase with distance. Thus, the amount of time taken to process the foveal item had about an equal effect on all peripheral stimuli.

In contrast, 5-year-old children did not show any pronounced effects of condition-either in their overall data or in their data at $6^{\circ}$. Moreover, the 5-year-olds did not appear to make use of an automatic "fovea first" processing strategy. Thus, the presence of a second item in the fovea did not interfere significantly with the 5-year-olds' ability to identify the peripheral item. Again, the lack of a "fovea first" processing strategy for the 5 -year-olds was supported by the order of report data from the double-form report condition. For the 5- year-olds, the order in which the items were reported was essentially random: $46 \%$ of the correctly reported peripheral items were reported first.

In conclusion, the findings of this study replicated the many existing studies which indicate that a subject's ability to identify peripherally presented stimuli is impaired when the subject must also process some foveally presented stimulus item. Secondly, this study supported Mackworth's notion that this impairment occurs whether or not the foveally presented item must actually be reported. More significantly, this study suggested that the use of the label "tunnel vision" for the phenomenon may be somewhat misleading, since the presence of the foveal stimulus seemed to have an equal effect on all peripheral locations and did not really "restrict" the size of the effective visual field. This study further suggested that this interference by the foveal item was due to the automatic tendency of older subjects to process this foveal item first. Finally, it appeared that this automatic fovea-first processing strategy was somehow learned, as it was not present in children 5 years of age. Thus, the presence of the foveally presented stimulus item was found to have no significant effect upon the performance of the 5-year-old children studied.

\section{REFERENCES}

Holmes, D. L. The world to the side: A developmental study of the ability to process peripheral visual information. $\mathrm{PhD}$ thesis, Harvard University, 1972.

IKEDA, M., \& TAKEUCHI, T. Influence of foveal load on the functional visual field. Perception \& Psychophysics, 1975, 18. $255-260$.

LAKOWSKI, R., \& Aspinall, R. A. Static perimetry in young children. Vision Research, 1969, 9, 305-312.

Leibowitz, H. W., \& Appelle, S. The effect of a central task on luminance thresholds for peripherally presented stimuli. Human Factors, 1969, 11, 387-392.

Mackworth, N. H. Visual noise causes tunnel vision. Psychonomic Science, 1965, 3, 67-68.

MACKW ORTH, N. H., \& BRUNER, J. S. How adults and children search and recognize pictures. Human Development, 1970, 13, 149-177.

Miller. L. K. Eye-movement latency as a function of age, stimulus uncertainty and position in the visual field. Perceptual and Motor Skills, 1969, 28, 631-636.

Miller, L. K. Developmental differences in the field of view during tachistoscopic presentation. Child Development, 1971. 42. 1543-1551.

Webster, R. G., \& Haslerid, G. M. Influence on extreme peripheral vision of attention to a visual or auditory task. Journal of Experimental Psychology, 1964, 68, 269-272.

Whiteside, J. A. Peripheral vision in children and adults. Child Development, 1976, 47, 290-293.

\section{NOTES}

1. The data from nine subjects were dropped after initial testing, from two adults because of extremely poor performance at the foveal distances and from four 5-year-olds and three 8-year-olds 
because of their difficulties in understanding the task and/or their short attention spans.

2. The following correction formula was utilized to eliminate any differences due to response biases. It takes into account the fact that subjects would be expected to get one (out of eight) responses correct by guessing alone.

$$
\text { Corrected score }=\text { Number correct }-\frac{\text { Number incorrect }}{\text { Number of alternatives }-1}
$$

Note that the double-form report condition was scored for accuracy on the peripheral item only.
3. Although the amount of variance declined systematically with age, this difference was not statistically reliable: $C(3,71)$ $=0.44, \mathrm{p}>.05$.

4. The use of planned orthogonal comparisons is justified since, even though the three-way interaction was insignificant, specific predictions about the effects of age and condition at $6^{\circ}$ were made at the onset of the study. These a priori predictions were based on both theoretical considerations and results of previous research (e.g., Holmes, 1972).

(Received for publication April 14, 1977; revision accepted October 3, 1977.) 